\title{
Clinical Difference between Acute Appendicitis and Acute Right-Sided Colonic Diverticulitis
}

\author{
Ji Ho Song, ${ }^{1}$ Yong Won Kim $\mathbb{D},{ }^{1,2}$ Sanghun Lee, ${ }^{1,2}$ Han Ho Do, ${ }^{1}$ Jun Seok Seo, ${ }^{1}$ \\ Jeong Hun Lee, ${ }^{1}$ and Seung Chul Lee ${ }^{1}$ \\ ${ }^{1}$ Department of Emergency Medicine, Dongguk University Ilsan Hospital, Dongguk University College of Medicine, Goyang, \\ Republic of Korea \\ ${ }^{2}$ Department of Emergency Medicine, Kangwon National University College of Medicine, Chuncheon, Republic of Korea
}

Correspondence should be addressed to Yong Won Kim; chiefong@naver.com

Received 30 June 2020; Revised 29 July 2020; Accepted 6 August 2020; Published 1 September 2020

Academic Editor: Roberto Cirocchi

Copyright (c) 2020 Ji Ho Song et al. This is an open access article distributed under the Creative Commons Attribution License, which permits unrestricted use, distribution, and reproduction in any medium, provided the original work is properly cited.

Background. Clinical presentations of acute appendicitis (AA) and acute right-sided colonic diverticulitis (ARCD) are similar. However, the usual treatment for each disease differs between surgical and conservative management. The aim of this study was to identify clinical differences between AA and ARCD. Method. We performed a single-center retrospective study on adult patients, with uncomplicated AA and ARCD confirmed by computed tomography, who visited an emergency department between March 2018 and August 2019. Clinical variables including past medical history, presented symptoms and signs, and laboratory findings were compared between the two groups. A logistic regression analysis was subsequently performed to differentiate ARCD from AA based on results of univariate analyses. Results. A total of 212 (79.1\%) and 56 (20.9\%) patients were enrolled in AA and ARSD groups, respectively. Logistic regression analysis revealed that a past history of diverticulitis [OR: 102.679 (95\% CI: 9.964-1058.055), $p<0.001$ ] was associated with ARCD, while ketonuria [OR: 2.907 (95\% CI: 1.091-7.745), $p=0.033$ ], anorexia [OR: 21.544 (95\% CI: 3.905-118.868), $p<0.001$ ], and neutrophilia [OR: 3.406 (95\% CI: 1.243-9.336), $p=0.017$ ] were associated with AA. Conclusion. Anorexia, neutrophilia, and ketonuria were predictors of AA while a history of diverticulitis was a predictor of ARCD.

\section{Introduction}

Acute appendicitis (AA) and acute right-sided colonic diverticulitis (ARCD) are inflammatory diseases that occur near anatomical locations. Their clinical presentations are similar, making it difficult to differentiate between them [1-3]. The current standard treatment for each disease is different. Appendectomy may be needed for AA while nonoperative conservative treatments such as bowel rest and/or antibiotics are usually used for uncomplicated diverticulitis [4-7]. Nevertheless, ARCD is often misdiagnosed as AA and revealed during surgery $[3,8]$. Computer tomography $(\mathrm{CT})$ can be used as a diagnostic imaging tool to avoid unnecessary surgical exploration or for ARCD mimicking AA [9]. However, it has the disadvantages of radiation exposure and cost. Although ultrasound is an alternative image modality to identify these two diseases without radiation hazard, it requires skillful users $[10,11]$. Furthermore, episodes of diverticulitis are reported to recur in about $30 \%$ of cases. Abdominal image testing for each episode to differentiate between these two diseases can be a burden for the patient $[6,12]$. In this respect, only a few studies have verified clinical differences between these two diseases $[13,14]$. The purpose of this study was to identify different factors initially presented in the emergency room between AA and ARCD.

\section{Materials and Methods}

2.1. Study Design and Population. This single-center retrospective review study was conducted on consecutive Asian patients with age of 18 years old or older who visited the emergency department of a tertiary university hospital between March 2018 and August 2019 in the Republic of Korea, and diagnosis of AA or acute colonic diverticulitis was 
confirmed by a radiologist through an abdominal CT scan. Exclusion criteria were as follows: (1) patients had complications resulting from appendicitis and diverticulitis verified by CT scan (such as perforation, abscess, and/or fistula), (2) diverticulitis was not included in the right side of the colon, and (3) patients were transferred to another facility.

2.2. Study Variables. Clinical data obtained from electronic medical records included age, sex, body mass index (BMI), the time from symptom onset to visiting the emergency room, past medical history of previous diverticulitis or chronic diseases (diabetes, hypertension), gastrointestinal symptoms and signs (right lower quadrant [RLQ] pain, migrated pain to the RLQ, anorexia, nausea and vomiting, diarrhea), physical exams (initial body temperature, RLQ tenderness, rebound tenderness), laboratory findings (complete blood count, serum alanine transaminase $[\mathrm{ALT}]$, serum creatinine $[\mathrm{Cr}]$, serum C-reactive protein [CRP], urine ketone), Alvarado score and its components, and outcomes (need for hospitalization, length of stay, need for surgical treatment, mortality).

2.3. Study Definition. ARCD was defined as diverticulitis originating from primary inflammation of diverticulosis sited at the cecum or ascending colon. A history of diverticulitis was defined if patients were previously diagnosed with any sited colonic diverticulitis before the study period. Anorexia was defined as loss of appetite for food. Elevated ALT was defined as serum ALT over the upper limit of the normal value of $33 \mathrm{IU} / \mathrm{L}$ for males or $25 \mathrm{IU} / \mathrm{L}$ for females [15]. Elevated $\mathrm{Cr}$ was defined as serum $\mathrm{Cr}$ over the upper limit of the normal value of $1.29 \mathrm{mg} / \mathrm{dL}$ for males or $1.1 \mathrm{mg} /$ $\mathrm{dL}$ for females [16]. Elevated CRP was defined as serum CRP concentration over $1.0 \mathrm{mg} / \mathrm{dL}$, indicating significant inflammation [17]. Ketonuria was defined by a positive urine ketone dipstick test. The Alvarado score, a 10-point clinical scoring system, was calculated by adding each score to each clinical factor listed in Table 1 [18].

2.4. Statistical Analysis. Study variables of AA and ARCD groups were compared. Continuous variables are presented as median values (interquartile range, IQR). They were compared with the Mann-Whitney test. Nominal data were calculated as percentages based on the frequency of occurrence and compared using Chi-squared or Fisher's exact test as appropriate. Multivariate logistic regression was used to correlate single variables with ARCD. Resulting odds ratios (ORs) and 95\% confidence intervals (95\% CIs) are presented. A two-sided $p$ value of less than 0.05 was considered statistically significant. All statistical analyses were performed using IBM Statistical Package for the Social Sciences (SPSS) software version 24.0 (SPSS, Inc., Chicago, IL, USA).

\section{Results}

During the study period, a total of 231 cases of acute appendicitis and 74 cases of acute colonic diverticulitis cases were diagnosed by abdominal CT scans of adult patients
TABLE 1: Alvarado score.

\begin{tabular}{lc}
\hline Components & Score \\
\hline Anorexia & 1 \\
Nausea or vomiting & 1 \\
Migrating pain to RLQ & 1 \\
RLQ tenderness & 1 \\
Rebound tenderness & 2 \\
Elevated temperature $\left(>37.3^{\circ}\right)$ & 1 \\
Leukocytosis $>10,000 / \mathrm{mm}^{3}$ & 2 \\
Neutrophilia $>75 \%$ & 1 \\
Total & 10 \\
\hline
\end{tabular}

RLQ: right lower quadrant.

admitted to the emergency department. Among them, complicated cases (12 cases of appendicitis and 3 cases of diverticulitis), cases with non-right-side diverticulitis $(n=14)$, and cases that were transferred out ( 7 cases of acute appendicitis, 1 case of acute colonic diverticulitis) were excluded. Finally, 212 (79.1\%) and 56 (20.9\%) patients were enrolled in AA and ARCD groups, respectively.

Patient characteristics including clinical factors associated with each disease group and outcome are shown in Table 2 . The AA group had more RLQ pain $(94.3 \%$ vs. $85.7 \%$, $p=0.042)$, ketonuria ( $40.0 \%$ vs. $21.3 \%, p=0.018)$, anorexia ( $35.8 \%$ vs. $7.1 \%, p<0.001)$, RLQ tenderness $(98.1 \%$ vs. $87.5 \%$, $p=0.002)$, rebound tenderness ( $40.1 \%$ vs. $25.0 \%, p=0.043$ ), neutrophilia $(62.7 \%$ vs. $37.5 \%, p<0.001)$, admission care (100\% vs. $42.9 \%, p<0.001$ ), and surgical treatment ( $99.1 \%$ vs. $0 \%, p<0.001)$ than the ARCD group. The AA group had higher percentage of neutrophils [78.6 (70.2-84.8) vs. 73.6 (67.1-77.0), $p<0.001$ ] and Alvarado scores [6 (4-7) vs. 5 (3-6), $p<0.001]$ than the ARCD group. The ARCD group had longer onset-to-visit intervals [24 (13-48) vs. 11 (3-24) hours, $p<0.001$ ], more past histories of diverticulitis $(23.2 \%$ vs. $0.9 \%, p<0.001)$, and higher percentage of lymphocytes [18.2 (13.8-24.9) vs. $14.1(9.3-22.0), p=0.003]$ than the AA group. There was no significant difference in other single clinical variables between the two groups.

Multivariate analysis revealed that factors predictive of ARCD were past history of diverticulitis [OR: 102.679 (95\% CI: 9.964-1058.055), $p<0.001$ ], anorexia [OR: $0.046(95 \%$ CI: 0.008-0.256), $p<0.001$ ], ketonuria [OR: 0.344 (95\% CI: $0.129-0.961), p=0.033$ ], and neutrophilia [OR: $0.294(95 \%$ CI: 0.107-0.805), $p=0.017$ ] (Table 3).

\section{Discussion}

Diverticulosis affects approximately $25.1 \%$ of Asian population. It accounts for $87.9 \%$ of colonic diverticulosis cases involving the right side. This percentage is significantly higher than that in Western countries [19]. ARCD has been reported to occur at a relatively young age. The overall prevalence of diverticulitis is $75 \%$ in Asian population $[20,21]$. This study was limited to Asians. In the present study, $81.1 \%$ of colonic diverticulitis cases were ARCD cases. Our results showed that more surgical treatment was provided for AA, whereas conservative management was more provided for ARCD, consistent with a previous study 
TAвLE 2: General characteristics.

\begin{tabular}{|c|c|c|c|c|}
\hline Parameters & Total $N=268$ & AA group $N=212(79.1 \%)$ & ARCD group $N=56(20.9 \%)$ & $p$ value \\
\hline Age (yr) & $40(31-52)^{*}$ & $39(30-53)^{*}$ & $41(33-47)^{*}$ & 0.224 \\
\hline Male gender, no. (\%) & $131(48.9)$ & $107(50.5)^{*}$ & $24(42.9)^{*}$ & 0.368 \\
\hline BMI $\left(\mathrm{kg} / \mathrm{m}^{2}\right)$ & $23.3(20.7-25.9)^{*}$ & $23.4(20.9-25.9)^{*}$ & $22.4(20.5-25.9)^{*}$ & 0.389 \\
\hline Onset-to-visit interval (hr) & $15(4-47)^{*}$ & $11(3-24)^{*}$ & $24(13-48)^{*}$ & $<0.001$ \\
\hline \multicolumn{5}{|l|}{ Past medical history } \\
\hline Diabetes, no. (\%) & $11(4.1)$ & $8(3.8)$ & $3(5.4)$ & 0.704 \\
\hline \multirow{2}{*}{$\begin{array}{l}\text { Hypertension, no. (\%) } \\
\text { History of diverticulitis, } \\
\text { no. (\%) }\end{array}$} & $18(6.7)$ & $12(5.7)$ & $6(10.7)$ & 0.226 \\
\hline & $15(5.6)$ & $2(0.9)$ & $13(23.2)$ & $<0.001$ \\
\hline \multicolumn{5}{|l|}{ Symptoms and signs } \\
\hline Body temperature $\left({ }^{\circ} \mathrm{C}\right)$ & $36.8(36.5-37.3)^{*}$ & $36.8(36.5-37.3)^{*}$ & $36.8(36.5-37.1)^{*}$ & 0.432 \\
\hline RLQ pain, no. (\%) & $248(92.5)$ & $200(94.3)$ & $48(85.7)$ & 0.042 \\
\hline Diarrhea, no. (\%) & $48(17.9)$ & $34(16.0)$ & $14(25.0)$ & 0.169 \\
\hline Constipation, no. (\%) & $8(3.0)$ & $4(1.9)$ & $4(7.1)$ & 0.062 \\
\hline \multicolumn{5}{|l|}{ Laboratory findings } \\
\hline WBC & $11,470(9,110-14,270)^{*}$ & $11,740(9,155-14,550)^{*}$ & $11,160(8,920-12,900)^{*}$ & 0.224 \\
\hline $\begin{array}{l}\text { Absolute neutrophil } \\
\text { count }\end{array}$ & $8,860(6,340-11,670)^{*}$ & $9,210(6,410-11,830)^{*}$ & $7,960(6,130-9,930)^{*}$ & 0.063 \\
\hline Neutrophil (\%) & $77.5(69.1-83.3)^{*}$ & $78.6(70.2-84.8)^{*}$ & $73.6(67.1-77.0)^{*}$ & $<0.001$ \\
\hline Lymphocyte (\%) & $14.8(10.5-22.9)^{*}$ & $14.1(9.3-22.0)^{*}$ & $18.2(13.8-24.9)^{*}$ & 0.003 \\
\hline $\mathrm{Hb}(\mathrm{g} / \mathrm{dL})$ & $13.9(12.7-14.9)^{*}$ & $14.0(12.8-15.0)^{*}$ & $13.8(12.3-14.7)^{*}$ & 0.374 \\
\hline Hct $(\%)$ & $40.8(37.5-43.9)^{*}$ & $40.8(37.7-43.9)^{*}$ & $40.7(36.9-43.8)^{*}$ & 0.920 \\
\hline Elevated ALT, no. (\%) & $36(13.4)$ & $31(14.6)$ & $5(8.9)$ & 0.286 \\
\hline Elevated Cr, no. (\%) & $6(2.2)$ & $5(2.4)$ & $1(1.8)$ & 1.000 \\
\hline Elevated CRP, no. (\%) & $155(57.1)$ & $123(58.0)$ & $32(57.1)$ & 1.000 \\
\hline $\mathrm{CRP}(\mathrm{mg} / \mathrm{dL})$ & $1.5(0.4-4.6)^{*}$ & $1.4(0.3-4.2)^{*}$ & $2.2(0.8-4.6)^{*}$ & 0.138 \\
\hline Ketonuria, no. (\%) & $90 / 247(36.4)$ & $80 / 200(40.0)$ & $10 / 47(21.3)$ & 0.018 \\
\hline \multicolumn{5}{|c|}{ Alvarado score and components } \\
\hline Alvarado score & $6(4-7)^{*}$ & $6(4-7)$ & $5(3-6)^{*}$ & $<0.001$ \\
\hline Migration pain, no. (\%) & $49(18.3)$ & $44(20.8)$ & $5(8.9)$ & 0.051 \\
\hline Anorexia, no. (\%) & $90(33.6)$ & $76(35.8)$ & $4(7.1)$ & $<0.001$ \\
\hline $\begin{array}{l}\text { Nausea or vomiting, no. } \\
(\%)\end{array}$ & $92(32.7)$ & $78(35.1)$ & $14(23.7)$ & 0.153 \\
\hline RLQ tenderness, no. (\%) & 257 (95.9) & $208(98.1)$ & $49(87.5)$ & 0.002 \\
\hline $\begin{array}{l}\text { Rebound tenderness, } \\
\text { no. (\%) }\end{array}$ & $99(36.9)$ & $85(40.1)$ & $14(25.0)$ & 0.043 \\
\hline $\begin{array}{l}\text { Body temperature } \\
\geq 37.3^{\circ} \mathrm{C} \text {, no. }(\%)\end{array}$ & $71(26.5)$ & $59(27.8)$ & $12(21.4)$ & 0.396 \\
\hline Leukocytosis, no. (\%) & $179(66.8)$ & $142(67.0)$ & $37(66.1)$ & 1.000 \\
\hline Neutrophilia, no. (\%) & $154(57.5)$ & $133(62.7)$ & $21(37.5)$ & 0.001 \\
\hline \multicolumn{5}{|l|}{ Outcomes } \\
\hline Admission care, no. (\%) & $236(88.1)$ & $212(100)$ & $24(42.9)$ & $<0.001$ \\
\hline Hospital days & $5(4-6)^{*}$ & $5(4-6)^{*}$ & $5(4-6)^{*}$ & 0.574 \\
\hline $\begin{array}{l}\text { Surgical treatment, no. } \\
(\%)\end{array}$ & $211(78.7)$ & $210(99.1)$ & 0 & $<0.001$ \\
\hline Mortality, no. (\%) & $1(0.4)$ & $1(0.5)$ & 0 & 1.000 \\
\hline
\end{tabular}

${ }^{*}$ Median (interquartile range); AA: acute appendicitis; ARCD: acute right-sided colonic diverticulitis; BMI: body mass index; RLQ: right lower quadrant; WBC: white blood cell; Hb: hemoglobin; Hct: hematocrit; LDH: lactate dehydrogenase; ALT: alanine transaminase; Cr: creatinine; CRP: C-reactive protein.

TABLE 3: Multivariate analysis of predictors of ARCD compared to AA.

\begin{tabular}{lccc}
\hline Predictors of acute ARCD & Odds ratio & $95 \%$ CI & $p$ value \\
\hline Onset-to-visit interval (hr) & 1.000 & $0.999-1.001$ & 0.800 \\
History of diverticulitis & 102.679 & $9.964-1058.055$ & $<0.001$ \\
Anorexia & 0.046 & $0.008-0.256$ & $<0.001$ \\
RLQ pain with tenderness & 0.311 & $0.094-1.029$ & 0.056 \\
Rebound tenderness & 0.455 & $0.182-1.138$ & 0.092 \\
Ketonuria & 0.344 & $0.129-0.916$ & 0.033 \\
Neutrophilia & 0.294 & $0.107-0.805$ & 0.017 \\
\hline
\end{tabular}

CI: confidence interval; ARCD: acute right-sided colonic diverticulitis; AA: acute appendicitis; RLQ: right lower quadrant. 
[7]. Therefore, distinguishing these two diseases will be important for determining a therapeutic plan and avoiding unnecessary surgery for ARCD patients due to misdiagnosis or presumptive diagnosis as AA. The Alvarado scoring system is a classical tool for distinguishing appendicitis from other abdominal diseases [18]. However, even appendicitis can show equivocal Alvarado scores. Some studies have revealed that ARCD patients have higher or broader range of Alvarado scores [22-24]. Therefore, it may not be enough to use this scoring system as a tool to distinguish between these diseases. Although Alvarado scores were different between the two groups in our single variable analysis (median value: 6 points for AA and 5 points for ARCD), it would be difficult to assign clinical meaning because scores of 5 to 6 have an equivocal probability for appendicitis [25]. Currently, few studies have reported clinical differences in symptoms and signs (such as longer symptom duration associated with ARCD and nausea or vomiting, anorexia, migration pain, and RLQ pain associated with AA) between $\mathrm{AA}$ and $\mathrm{ARCD}$ $[13,14,22]$. Most differences in symptoms might be due to different pathophysiologies and elapsing course of these two diseases. Although both diseases have similar final symptoms due to localized peritonitis, appendicitis has a sequential reaction with prodromal symptoms due to blockage and dilatation of the appendix first. The increase in intraluminal pressure then results in wall necrosis [26]. However, each subjective indicator might have a risk of bias by clinicians or patients. Otherwise, a few studies have reported that some laboratory factors (such as neutrophilia and high CRP) are associated with AA rather than ARCD $[13,14]$. Like results of most previous studies, we found that a previous history of diverticulitis was a predictor of ARCD while anorexia and neutrophilia were predictors of AA.

An insufficient number of studies have reported the usefulness of leukocytosis for differentiating between the two diseases and, thus, it remains controversial [22, 27]. Shin et al. [13] have reported an elevated proportion of lymphocytes and a near-normal proportion of segmented neutrophils in ARCD. However, no hypothesis has been suggested to explain this phenomenon. Our results supported the relationship between neutrophilia and AA, and higher fractions of lymphocytes were also related to ARCD. Sasaki et al. [14] have categorized high serum CRP as $>3.0 \mathrm{mg} / \mathrm{dL}$ and reported that high serum CRP is associated with ARCD. However, our results did not support such finding. There was no statistical difference in the percentage of high serum CRP ( $32.5 \%$ vs. $37.5 \%, p=0.526)$ in our study between AA and ARCD groups, even when serum CRP values of over $3.0 \mathrm{mg} / \mathrm{dL}$ were used as criteria. Serum CRP is known to peak after 48 hours due to its response to inflammation [28]. Our study and the study by Sasaki et al. [14] differed in the time interval from onset-tovisit in the ARCD patients ( $24 \mathrm{~h}$ vs. $48 \mathrm{~h}$ ). This could affect blood sample collection time after infection. Therefore, it might be inappropriate to compare these two studies. Moreover, it has been revealed that serum CRP levels will increase over time in appendicitis, raising question as to whether high CRP levels are more relevant to ARCD than to

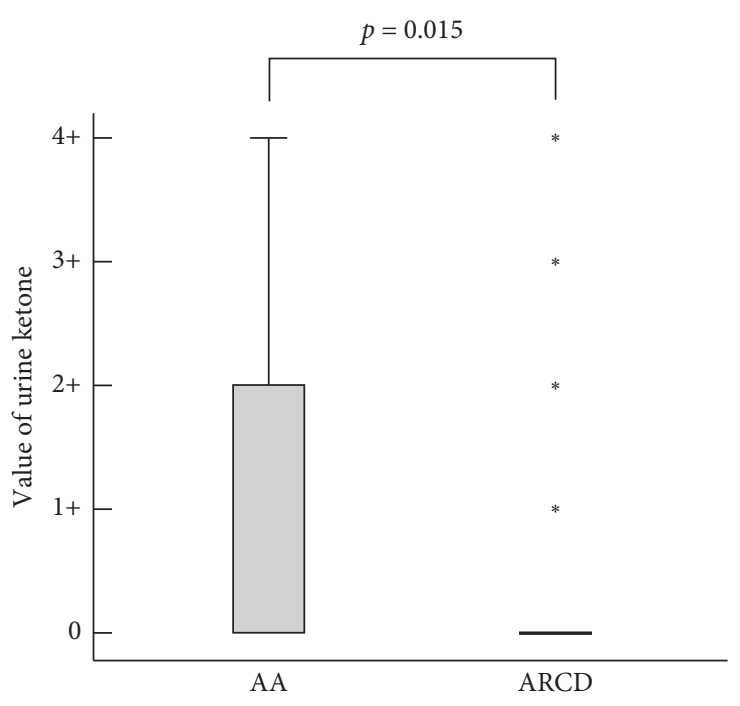

FIGURE 1: Boxplot showing urine ketone values from dipstick tests for acute appendicitis and acute right-sided colonic diverticulitis patients.

AA if confounding time factors related to CRP increase are not removed [29].

Our results revealed that ketonuria was one of the objective predictors of AA. We presented a boxplot for the distribution of urine ketone values between the two groups as shown in Figure 1. A few studies reported that ketonuria was seen in $12 \%$ to $45 \%$ of the AA cases, but there have been no comparative studies between AA and ARCD [30, 31]. Ketones are known as end-products of fatty acid metabolism. Ketonuria indicates that the body is excessively using fat over carbohydrates as the major source of energy [32]. Ketonuria could arise from dietary conditions (such as fasting, nausea and vomiting, and anorexia) and metabolic conditions (including type-1 diabetes, fever, and pregnancy) [33]. In our study, there were no patients with type-1 diabetes or pregnant women. There was no significant difference in body temperature between the two groups either. Therefore, the probable cause of ketonuria in appendicitis might be related to dietary conditions such as anorexia because these conditions were found more in the AA group than in the ARCD group. When we made a simple rule for predicting ARCD that included objective clinical factors such as no ketonuria and no neutrophilia with the presence of a history of diverticulitis, the calculated positive predictive value (PPV) was $87.5 \%$ (95\% CI: $46.7-98.2 \%$ ), the negative predictive value (NPV) was 95.2\% (95\% CI: 94.6-95.7\%), and the specificity was $99.8 \%$ (95\% CI: $99.3-99.9 \%)$. This rule might be a useful tool for distinguishing ARCD from AA.

This study has some limitations. First, this study was conducted on an Asian population at a single center in the Republic of Korea. Therefore, results of this study might not represent all races and nations. However, the presentation of diverticulitis in the Asian population is unusual because it most commonly involves the right side of the colon. The exact pathological mechanism of diverticular disease is 
unclear, although several theories related to genetics, diet, motility, and microbiome that might be affected by individual races and cultures have been presented [34]. Therefore, this study has some clinical implications because not many studies have been reported in an Asian population.

Second, because this study was a retrospective study rather than a confirmative study, data or cases might be missing. Specifically, for subjective symptoms and signs, it was difficult to accurately describe the intensity or presence without a proper prospective protocol.

Third, ketonuria is roughly correlated with serum ketone concentrations, but the absence of ketonuria does not mean the absence of blood ketone bodies. However, we could not obtain the serum ketone concentrations to distinguish between AA and ARCD as that analysis was not included in the routine ER blood tests because it is more expensive than urine analysis and has not been proven useful in the diagnosis of these diseases. Further studies that quantitatively analyze these factors are needed.

\section{Conclusion}

Our results suggest that anorexia, neutrophilia, and ketonuria are useful predictors of AA, but not ARCD. Conversely, a history of diverticulitis was a useful predictor of ARCD, but not AA. If a history of diverticulitis is present without neutrophilia or ketonuria, then the PPV is $87.5 \%$ and the specificity is $99.8 \%$ for ARCD. Our findings could be used for differential diagnosis between AA and ARCD to reduce unnecessary additional imaging studies for ARCD.

\section{Data Availability}

All datasets used and/or analyzed in the current study are available from the corresponding author upon reasonable request.

\section{Ethical Approval}

This study was approved by the Institutional Review Board (IRB) of Dongguk University Ilsan Hospital, Dongguk University (2018-11-007). Informed consent was waived by the IRB.

\section{Conflicts of Interest}

The authors declare that they have no conflicts of interest regarding the publication of this paper.

\section{References}

[1] O. Karatepe, O. Gulcicek, G. Adas et al., "Cecal diverticulitis mimicking acute Appendicitis: a report of 4 cases," World Journal of Emergency Surgery, vol. 3, no. 1, p. 16, 2008.

[2] C. Y. Lo and K. W. Chu, "Acute diverticulitis of the right colon," The American Journal of Surgery, vol. 171, no. 2, pp. 244-246, 1996.

[3] J. S. Lane, R. Sarkar, P. J. Schmit, C. F. Chandler, and J. E. Thompson, "Surgical approach to cecal diverticulitis," Journal of the American College of Surgeons, vol. 188, no. 6, pp. 629-634, 1999.
[4] D. L. H. Baird, C. Simillis, C. Kontovounisios, S. Rasheed, and P. P. Tekkis, "Acute appendicitis," BMJ, vol. 357, p. j1703, 2017.

[5] R. Detry, J. Jamez, A. Kartheuser et al., "Acute localized diverticulitis: optimum management requires accurate staging," International Journal of Colorectal Disease, vol. 7, no. 1, pp. 38-42, 1992.

[6] M. M. Szojda, M. A. Cuesta, C. M. Mulder, and R. J. F. Feltbersma, "Review article: management of diverticulitis," Alimentary Pharmacology \& Therapeutics, vol. 26, pp. 67-76, 2007.

[7] K. Matsushima, "Management of right-sided diverticulitis: a retrospective review from a hospital in Japan," Surgery Today, vol. 40, no. 4, pp. 321-325, 2010.

[8] A. Sardi, A. Gokli, and J. A. Singer, "Diverticular disease of the cecum and ascending colon. a review of 881 cases," The American Surgeon, vol. 53, no. 1, pp. 41-45, 1987.

[9] B. A. Birnbaum and E. J. Balthazar, "CT of appendicitis and diverticulitis," Radiologic Clinics of North America, vol. 32, no. 5, pp. 885-898, 1994.

[10] Y.-H. Chou, H.-J. Chiou, C.-M. Tiu et al., "Sonography of acute right side colonic diverticulitis," The American Journal of Surgery, vol. 181, no. 2, pp. 122-127, 2001.

[11] P. Rossi, P. Covarelli, F. Mosci, R. Bisacci, B. Sensi, and L. Moggi, "Ultrasonography in the management of acute appendicitis," Surgical Endoscopy, vol. 10, no. 6, pp. 619-621, 1996.

[12] N. Stollman and J. B. Raskin, "Diverticular disease of the colon," The Lancet, vol. 363, no. 9409, pp. 631-639, 2004.

[13] J.-H. Shin, B.-H. Son, and H. Kim, "Clinically distinguishing between appendicitis and right-sided colonic diverticulitis at initial presentation," Yonsei Medical Journal, vol. 48, no. 3, pp. 511-516, 2007.

[14] Y. Sasaki, F. Komatsu, N. Kashima et al., "Clinical differentiation of acute appendicitis and right colonic diverticulitis: a case-control study," World Journal of Clinical Cases, vol. 7, no. 12, pp. 1393-1402, 2019.

[15] J. K. Lee, J. H. Shim, H. C. Lee et al., "Estimation of the healthy upper limits for serum alanine aminotransferase in Asian populations with normal liver histology," Hepatology, vol. 51, no. 5, pp. 1577-1583, 2010.

[16] C. Jones, G. McQuillan, J. Kusek et al., "Serum creatinine levels in the US population: third national health and nutrition examination survey," American Journal of Kidney Diseases, vol. 32, no. 6, pp. 992-999, 1998.

[17] I. Kushner and M. J. Antonelli, "What should we regard as an "elevated" C-reactive protein level?" Annals of Internal Medicine, vol. 163, no. 4, p. 326, 2015.

[18] A. Alvarado, "A practical score for the early diagnosis of acute appendicitis," Annals of Emergency Medicine, vol. 15, no. 5, pp. 557-564, 1986.

[19] C. C. Chan, K. L. Lo, E. C. H. Chung, S. S. Lo, and T. Y. W. Hon, "Colonic diverticulosis in Hong Kong: distribution pattern and clinical significance," Clinical Radiology, vol. 53, no. 11, pp. 842-844, 1998.

[20] L. E. Hughes, "Postmortem survey of diverticular disease of the colon. I. Diverticulosis and diverticulitis," Gut, vol. 10, no. 5, pp. 336-344, 1969.

[21] J.-K. Park, J.-K. Sung, S.-H. Choi, S.-S. Yun, and S. Lee, "Clinical analysis of right colon diverticulitis," Annals of Surgical Treatment and Research, vol. 64, no. 1, pp. 44-48, 2003.

[22] S. C. Chen, K. J. Chang, T. C. Wei, S. C. Yu, and S. M. Wang, "Can cecal diverticulitis be differentiated from acute 
appendicitis?" Journal of the Formosan Medical Association, vol. 93, no. 3, pp. 263-265, 1994.

[23] F. Monari, M. Cervellera, B. Pirrera et al., "Right-sided acute diverticulitis: a single Western center experience," International Journal of Surgery, vol. 44, pp. 128-131, 2017.

[24] R. McKay and J. Shepherd, "The use of the clinical scoring system by Alvarado in the decision to perform computed tomography for acute appendicitis in the ED," The American Journal of Emergency Medicine, vol. 25, no. 5, pp. 489-493, 2007.

[25] C. D. Douglas, N. E. Macpherson, P. M. Davidson, and J. S. Gani, "Randomised controlled trial of ultrasonography in diagnosis of acute appendicitis, incorporating the Alvarado score," BMJ, vol. 321, no. 7266, p. 919, 2000.

[26] J. M. Wagner, W. P. McKinney, and J. L. Carpenter, "Does this patient have appendicitis?" JAMA: The Journal of the American Medical Association, vol. 276, no. 19, pp. 1589-1594, 1996.

[27] N. S. Painter, "The cause of diverticular disease of the colon, its symptoms and its complications. Review and hypothesis," Journal of the Royal College of Surgeons of Edinburgh, vol. 30, no. 2, pp. 118-122, 1985.

[28] M. B. Pepys and G. M. Hirschfield, "C-reactive protein: a critical update," Journal of Clinical Investigation, vol. 111, no. 12, pp. 1805-1812, 2003

[29] H.-P. Wu, C.-Y. Lin, C.-F. Chang, Y.-J. Chang, and C.-Y. Huang, "Predictive value of C-reactive protein at different cutoff levels in acute appendicitis," The American Journal of Emergency Medicine, vol. 23, no. 4, pp. 449-453, 2005.

[30] C.-Y. Chen, L.-L. Zhao, Y.-R. Lin, K.-H. Wu, and H.-P. Wu, "Different urinalysis appearances in children with simple and perforated appendicitis," The American Journal of Emergency Medicine, vol. 31, no. 11, pp. 1560-1563, 2013.

[31] F. K. Althoubaity, "Suspected acute appendicitis in female patients," Saudi Medical Journal, vol. 27, no. 11, pp. 16671673, 2006.

[32] L. Laffel, "Ketone bodies: a review of physiology, pathophysiology and application of monitoring to diabetes," Diabetes/Metabolism Research and Reviews, vol. 15, no. 6, pp. 412-426, 1999.

[33] J. A. Simerville, W. C. Maxted, and J. J. Pahira, "Urinalysis: a comprehensive review," American Family Physician, vol. 71, no. 6, pp. 1153-1162, 2005.

[34] L. L. Strate, R. Modi, E. Cohen, and B. M. R. Spiegel, "Diverticular disease as a chronic illness: evolving epidemiologic and clinical insights," American Journal of Gastroenterology, vol. 107, no. 10, pp. 1486-1493, 2012. 